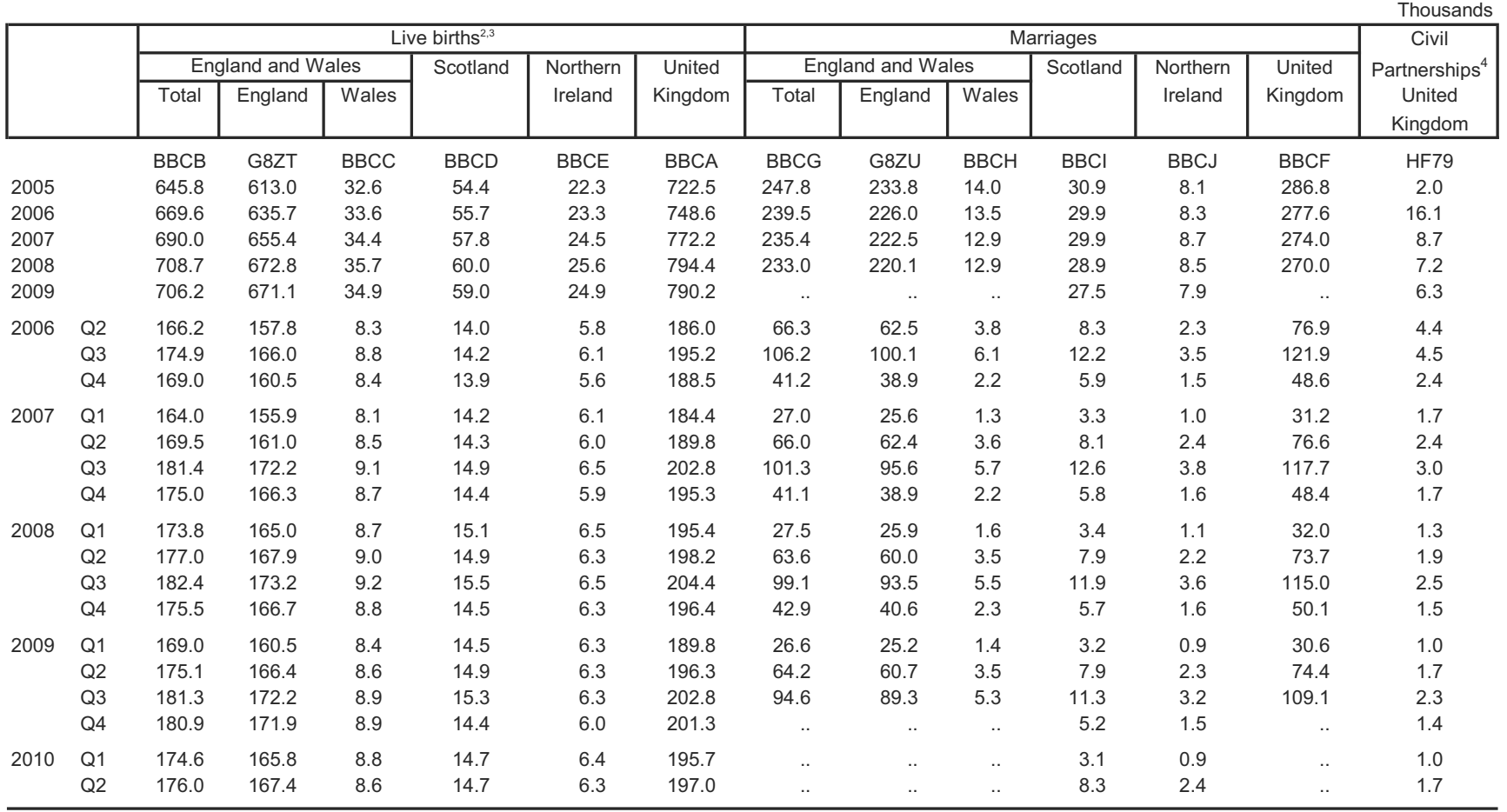

Note: Figures may not add exactly due to rounding. Any data in red is provisional.

1 Excluding stillbirths.

2 Birth figures for England and also for Wales each exclude events for persons usually resident outside England and Wales. These events are, however, included in the totals for England and Wales combined and for the United Kingdom.

3 For England and Wales, figures relate to numbers occurring in a period; for Scotland and Northern Ireland, figures relate to those registered in a period. 4 The Civil Partnership Act 2004 came into force on 5 December 2005 in the UK.

Sources: Office for National Statistics; Health Statistics Quarterly No. 48); General Register Office for Scotland; Northern Ireland Statistics \& Research Agency.

\begin{tabular}{|c|c|c|c|c|c|c|c|c|c|c|c|}
\hline \multicolumn{2}{|c|}{ Table 8.2} & \multicolumn{10}{|c|}{ Deaths registered } \\
\hline & & & & & & & & & & & Thousands \\
\hline & & \multicolumn{5}{|c|}{ Total } & \multicolumn{5}{|c|}{ Infants aged under one year } \\
\hline & & \multicolumn{2}{|c|}{ England and Wales } & \multirow[t]{2}{*}{ Scotland } & \multirow{2}{*}{$\begin{array}{c}\text { Northern } \\
\text { Ireland } \\
\end{array}$} & \multirow{2}{*}{$\begin{array}{c}\text { United } \\
\text { Kingdom }\end{array}$} & \multicolumn{2}{|c|}{ England and Wales } & \multirow[t]{2}{*}{ Scotland } & \multirow{2}{*}{$\begin{array}{l}\text { Northern } \\
\text { Ireland }\end{array}$} & \multirow{2}{*}{$\begin{array}{c}\text { United } \\
\text { Kingdom }\end{array}$} \\
\hline & & Total & Wales & & & & Total & Wales & & & \\
\hline & & BBDB & BBDC & BBDD & BBDE & BBDA & BBDG & $\mathrm{BBDH}$ & BBDI & BBDJ & BBDF \\
\hline 2005 & & 513.0 & 32.2 & 55.7 & 14.2 & 583.0 & 3.25 & 0.14 & 0.28 & 0.14 & 3.67 \\
\hline 2006 & & 502.6 & 31.1 & 55.1 & 14.5 & 572.2 & 3.37 & 0.14 & 0.25 & 0.12 & 3.74 \\
\hline 2008 & & 509.1 & 32.1 & 55.7 & 14.9 & 579.7 & 3.37 & 0.15 & 0.25 & 0.12 & 3.75 \\
\hline $2009^{1}$ & & 491.3 & 31.0 & 53.9 & 14.4 & 559.6 & 3.31 & 0.17 & 0.24 & 0.13 & 3.68 \\
\hline \multirow[t]{4}{*}{2006} & Q1 & 141.0 & 8.7 & 14.9 & 4.0 & 159.9 & 0.82 & 0.03 & 0.05 & 0.03 & 0.90 \\
\hline & Q2 & 123.9 & 7.6 & 13.9 & 3.6 & 141.4 & 0.84 & 0.03 & 0.07 & 0.03 & 0.94 \\
\hline & Q3 & 114.6 & 7.2 & 12.7 & 3.4 & 130.7 & 0.85 & 0.04 & 0.05 & 0.03 & 0.93 \\
\hline & Q4 & 123.1 & 7.5 & 13.6 & 3.5 & 140.2 & 0.86 & 0.04 & 0.07 & 0.03 & 0.97 \\
\hline \multirow[t]{3}{*}{2007} & Q1 & 139.3 & 8.8 & 15.8 & 4.2 & 159.3 & 0.80 & 0.05 & 0.07 & 0.04 & 0.91 \\
\hline & Q2 & 121.0 & 7.9 & 13.4 & 3.6 & 138.0 & 0.88 & 0.04 & 0.08 & 0.03 & 0.99 \\
\hline & Q4 & 129.7 & 8.3 & 14.2 & 3.6 & 147.5 & 0.83 & 0.05 & 0.06 & 0.02 & 0.90 \\
\hline \multirow[t]{4}{*}{2008} & Q1 & 136.4 & 8.6 & 15.1 & 4.1 & 155.6 & 0.86 & 0.04 & 0.06 & 0.03 & 0.95 \\
\hline & Q2 & 123.1 & 7.7 & 13.5 & 3.6 & 140.2 & 0.79 & 0.04 & 0.06 & 0.03 & 0.88 \\
\hline & Q3 & 114.3 & 7.3 & 12.6 & 3.4 & 130.3 & 0.87 & 0.04 & 0.07 & 0.03 & 0.97 \\
\hline & Q4 & 135.3 & 8.5 & 14.5 & 3.7 & 153.5 & 0.85 & 0.04 & 0.06 & 0.04 & 0.95 \\
\hline \multirow[t]{4}{*}{2009} & $\mathrm{Q}^{1}{ }^{1}$ & 138.9 & 8.9 & 14.8 & 4.2 & 157.9 & 0.83 & 0.03 & 0.06 & 0.04 & 0.93 \\
\hline & Q21 & 115.7 & 7.3 & 12.8 & 3.4 & 131.9 & 0.85 & 0.04 & 0.06 & 0.03 & 0.94 \\
\hline & Q3 $^{1}$ & 110.7 & 6.9 & 12.4 & 3.2 & 126.4 & 0.80 & 0.05 & 0.06 & 0.04 & 0.89 \\
\hline & Q4 ${ }^{1}$ & 126.1 & 7.9 & 13.8 & 3.6 & 143.5 & 0.84 & 0.05 & 0.05 & 0.02 & 0.91 \\
\hline \multirow[t]{2}{*}{2010} & Q1 ${ }^{1}$ & 134.5 & 8.5 & 14.7 & 4.1 & 153.3 & 0.80 & 0.04 & 0.06 & 0.04 & 0.91 \\
\hline & Q21 & 117.3 & 7.4 & 12.8 & 3.3 & 133.4 & 0.80 & 0.03 & 0.05 & 0.04 & 0.88 \\
\hline
\end{tabular}

1 Provisional

Sources: General Register Office (Scotland); Northern Ireland Statistics and Research Agency; Office for National Statistics 


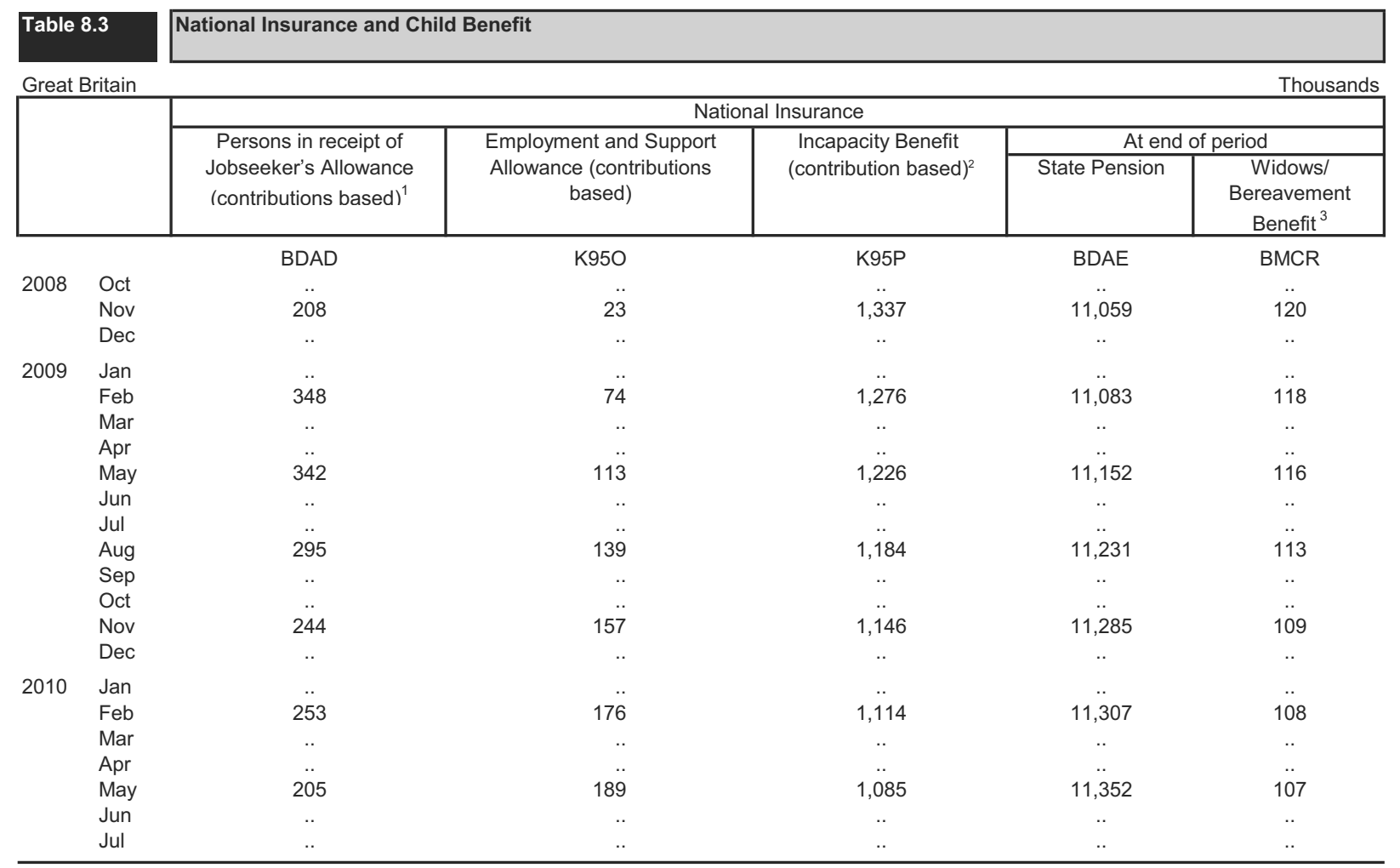

1 Jobseeker's Allowance figures have been derived by rating up to $5 \%$ sample to $100 \%$ counts. This figure is based on Contribution Based only Jobseekers Allowance and excludes those receiving contributory benefit with an amount of income based benefit.

2 Incapacity Benefit is contribution based, effectively making these figures Incapacity Benefit recipient counts. Claimants who are assessed as being incapable of work and do not meet the contribution conditions can receive 'IB Credits only' and are not included in the recipient count. Excludes overseas and Channel Island cases.

3 Includes all Widow's Benefit and Bereavement Benefit except Widow's Payment and Bereavement Payment. Excludes overseas and Channel Island cases.

Source: Department for Workand Pensions

\begin{tabular}{|c|c|c|c|c|}
\hline Tabld 8.4 & \multicolumn{4}{|c|}{ Child and Working Tax Credit $^{1}$} \\
\hline \multicolumn{4}{|c|}{ United Kingdom } & \multirow{3}{*}{$\begin{array}{c}\text { Thousands } \\
\text { No of children in these families }\end{array}$} \\
\hline & \multicolumn{3}{|c|}{ Families in work receiving credit: } & \\
\hline & All families ${ }^{2}$ & Two-adult families & One-adult families & \\
\hline & WMPT & WMPU & WMPV & WMPW \\
\hline $2006 \mathrm{Apr}$ & 4,601 & 3,307 & 1,294 & 7,446 \\
\hline 2006 Dec & 4,526 & 3,237 & 1,289 & 7,292 \\
\hline $2007 \mathrm{Apr}$ & 4,619 & 3,296 & 1,323 & 7,425 \\
\hline $2007 \mathrm{Dec}$ & 4,541 & 3,192 & 1,349 & 7,252 \\
\hline $2008 \mathrm{Apr}$ & 4,660 & 3,256 & 1,404 & 7,435 \\
\hline $2008 \mathrm{Dec}$ & 4,630 & 3,167 & 1,463 & 7,271 \\
\hline $2009 \mathrm{Apr}$ & 4,713 & 3,211 & 1,502 & 7,362 \\
\hline $2009 \mathrm{Dec}$ & 4712 & 3161 & 1551 & 7238 \\
\hline $2010 \mathrm{Apr}$ & 4,811 & 3,216 & 1,595 & 7,353 \\
\hline
\end{tabular}

1 For further information refer to Section 4 of the Annual Supplement in the January edition of the Monthly Digest.

2 Figures are separately rounded, which can lead to the components as shown not summing to the total.

Source: HM Revenue and Customs: 02071473067 


\begin{tabular}{|c|c|c|c|c|c|c|c|c|c|c|c|c|}
\hline \multicolumn{2}{|c|}{ Table 8.5} & \multicolumn{11}{|c|}{ Income Support/Pension Credit/Jobseeker's Allowance (income based) } \\
\hline \multicolumn{10}{|c|}{ Great Britain } & \multicolumn{3}{|c|}{ In a week in the month shown, thousands } \\
\hline & & \multicolumn{4}{|c|}{ Income supportt. } & \multirow{3}{*}{$\begin{array}{c}\text { Pension credit } \\
\text { All cases }\end{array}$} & \multirow{2}{*}{\multicolumn{3}{|c|}{$\begin{array}{l}\text { Employment and Support Allowance } \\
\text { (income based) }\end{array}$}} & \multirow{2}{*}{\multicolumn{3}{|c|}{$\begin{array}{l}\text { Jobseeker's Allowance } \\
\text { (income based) }\end{array}$}} \\
\hline & & \multirow{2}{*}{\begin{tabular}{|c|} 
Incapacity \\
benefits $^{3}$
\end{tabular}} & \multirow[t]{2}{*}{ Lone parents ${ }^{4}$} & \multirow[t]{2}{*}{ Others $^{5}$} & \multirow[t]{2}{*}{ All cases } & & & & & & & \\
\hline & & & & & & & $\begin{array}{c}\text { With } \\
\text { contibution } \\
\text { based benefit }\end{array}$ & $\begin{array}{c}\text { Without } \\
\text { contribution } \\
\text { based benefit }\end{array}$ & All cases & $\begin{array}{c}\text { With } \\
\text { contribution } \\
\text { based benefit }\end{array}$ & $\begin{array}{c}\text { Without } \\
\text { contribution } \\
\text { based benefit }\end{array}$ & All cases \\
\hline & & BAMD & BAME & BAMF & BAMG & A4EK & K95Q & K95R & K95S & DMUB & DMUC & DMUD \\
\hline \multirow{3}{*}{2006} & May & 1,183 & 775 & 157 & 2,115 & 2,717 & .. & .. & .. & 14 & 690 & 704 \\
\hline & Aug & 1,189 & 783 & 157 & 2,129 & 2,728 & .. & .. & .. & 13 & 695 & 708 \\
\hline & Nov & 1,193 & 776 & 164 & 2,132 & 2,739 & .. & .. & .. & 13 & 673 & 686 \\
\hline \multirow[t]{4}{*}{2007} & Feb & 1,188 & 771 & 165 & 2,124 & 2,731 & .. & .. & .. & 15 & 692 & 708 \\
\hline & May & 1,185 & 766 & 167 & 2,118 & 2,733 & .. & .. & .. & 12 & 628 & 640 \\
\hline & Aug & 1,187 & 764 & 167 & 2,118 & 2,736 & .. & .. & .. & 11 & 592 & 603 \\
\hline & Nov & 1,190 & 742 & 165 & 2,097 & 2,735 & .. & .. & .. & - & - & - \\
\hline \multirow[t]{4}{*}{2008} & Feb & 1,186 & 742 & 167 & 2,095 & 2,723 & .. & .. & .. & 12 & 592 & 604 \\
\hline & May & 1,183 & 739 & 170 & 2,092 & 2,719 & .. & .. & .. & 13 & 577 & 590 \\
\hline & Aug & 1,184 & 755 & 173 & 2,101 & 2,724 & .. & & .. & 15 & 623 & 638 \\
\hline & Nov & 1,178 & 729 & 173 & 2,080 & 2,731 & 2 & 23 & 25 & 19 & 706 & 725 \\
\hline \multirow[t]{4}{*}{2009} & Feb & 1,126 & 736 & 172 & 2,034 & 2,724 & 7 & 75 & 82 & 32 & 907 & 939 \\
\hline & May & 1,088 & 720 & 171 & 1,980 & 2,731 & 13 & 125 & 138 & 35 & 940 & 975 \\
\hline & Aug & 1,059 & 716 & 170 & 1,945 & 2,737 & 18 & 163 & 181 & 29 & 1,026 & 1,055 \\
\hline & Nov & 1,032 & 695 & 172 & 1,900 & 2,740 & 23 & 188 & 211 & 25 & 1,066 & 1,091 \\
\hline \multirow[t]{2}{*}{2010} & Feb & 1,009 & 692 & 174 & 1,875 & 2,735 & 28 & 213 & 240 & 26 & 1,107 & 1,133 \\
\hline & May & 989 & 679 & 175 & 1,843 & 2,734 & 33 & 239 & 272 & 21 & 1,011 & 1,032 \\
\hline
\end{tabular}

1 IS claimants have been assigned to a statistical group according to a hierarchy. The order is as shown in the table, i.e. "Incapacity Benefits",

"Lone Parents" etc. For example, a single parent claiming both Incapacity Benefits and Income Support will fall into the "Incapacity Benefits" category

2 Figures exclude "Minimum Income Guarantee" catergory.

3 "Incapacity Benefits" refer to claimants aged under 60 claiming Incapacity Benefit (IB) or Severe Disablement Allowance (SDA), including

IB credits only cases. This figure does not include Employment and Support Allowance claimants.

4 "Lone Parents" are single recipients of Income Support aged under 60 with a child under 16 who are not in receipt of IB/SDA.

5 "Others" are recipients of Income Support not in one of the other categories.

6 Since 6th October 2003, Income Support for claimants aged 60 or over have been paid via the new Pension Credit. Pension Credit eligibility

is also more generous than prior to 6 th October 2003 , increasing the numbers of pensioners in receipt.

7 JSA figures have been derived by rating up a $5 \%$ sample to $100 \%$ counts.

Source: Department for Work and Pensions

Table 8.6

Family health services

\begin{tabular}{|c|c|c|c|c|c|c|c|c|c|}
\hline & \multicolumn{6}{|c|}{ England and Wales } & \multicolumn{3}{|c|}{ Scotland } \\
\hline & \multicolumn{4}{|c|}{ Dental services } & \multicolumn{2}{|c|}{ Ophthalmic services ${ }^{1}$} & \multirow{3}{*}{$\begin{array}{c}\text { Dental services } \\
\text { Completed courses of } \\
\text { adult treatment and } \\
\text { cases of occasional } \\
\text { treatment }^{2,3} \\
\end{array}$} & \multicolumn{2}{|c|}{ Ophthalmic services } \\
\hline & \multicolumn{2}{|c|}{$\begin{array}{c}\text { Courses of treatments } \\
\text { delivered by } \\
\text { NHS Dentists }\end{array}$} & \multicolumn{2}{|c|}{$\begin{array}{c}\text { Patients seen } \\
\text { in previous } 24 \text { months } \\
\text { by NHS Dentists }\end{array}$} & \multirow[t]{2}{*}{$\begin{array}{c}\text { NHS } \\
\text { Sight tests }\end{array}$} & \multirow[t]{2}{*}{$\begin{array}{c}\text { NHS optical } \\
\text { voucher items } \\
\text { processed }\end{array}$} & & \multirow[t]{2}{*}{$\begin{array}{l}\text { Sight tests } \\
\text { paid for }\end{array}$} & \multirow{2}{*}{$\begin{array}{c}\text { Pairs of spectacles } \\
\text { paid for by SHBs } \\
\text { under the Voucher } \\
\text { Scheme } \\
\end{array}$} \\
\hline & England & Wales & England & Wales & & & & & \\
\hline \multicolumn{10}{|l|}{ 2006/07 } \\
\hline Apr-Jun & .. & 330 & 28,068 & 1,636 & & & .. & & \\
\hline Jul-Sept & .. & 499 & 28,084 & 1,631 & 5,659 & 2,051 & 708 & 386 & 106 \\
\hline Oct-Dec & .. & 557 & 28,076 & 1,637 & & & 737 & 384 & 108 \\
\hline Jan-Mar & & 573 & 28,098 & 1,643 & 5,501 & 1,996 & 742 & 432 & 116 \\
\hline \multicolumn{10}{|l|}{ 2007/08 } \\
\hline Apr-Jun & 9,051 & 548 & 27,879 & 1,625 & .. & .. & 753 & 413 & 117 \\
\hline Jul-Sept & 8,892 & 548 & 27,602 & 1,607 & 5,872 & 2,112 & 717 & 400 & 111 \\
\hline Oct-Dec & 8,909 & 553 & 27,264 & 1,596 & & & 736 & 390 & 109 \\
\hline Jan-Mar & 9,135 & 593 & 27,049 & 1,596 & 5,876 & 2,095 & 753 & 424 & 114 \\
\hline \multicolumn{10}{|l|}{$2008 / 09$} \\
\hline Apr-Jun & 9,323 & 579 & 26,935 & 1,599 & - & .. & 776 & 417 & 116 \\
\hline Jul-Sept & 9,277 & 585 & 27,033 & 1,614 & 6,005 & 2,214 & 761 & 430 & 116 \\
\hline Oct-Dec & 9,254 & 582 & 27,272 & 1,630 &.. & .. & 780 & 430 & 116 \\
\hline Jan-Mar & 9,572 & 605 & 27,502 & 1,643 & 5,993 & 2,193 & 789 & 451 & 120 \\
\hline \multicolumn{10}{|l|}{$2009 / 10$} \\
\hline Apr-Jun & 9,586 & .. & 27,656 & .. & .. & .. & 804 & 454 & 123 \\
\hline Jul-Sept & 9,623 & .. & 27,873 & .. & 6,426 & 2,403 & 800 & 439 & 116 \\
\hline Oct-Dec & 9,556 & 576 & 28,163 & 1,264 & .. & .. & 814 & 428 & 115 \\
\hline Jan-Mar & 9,825 & 585 & 28,363 & 1,278 & 6,121 & 2,298 & .. & 454 & 120 \\
\hline \multicolumn{10}{|l|}{$20010 / 11$} \\
\hline Apr-Jun & .. & .. & 28,521 & .. & .. & .. & 841 & 455 & 121 \\
\hline Jul-Sep & .. & & 28,703 & & & & 846 & 462 & 120 \\
\hline
\end{tabular}

1 Data on Ophthalmic Services are collected six-monthly and presented against the second quarter covered. From 2009/10 this information is only available annually.

2 Number scheduled for payment in the General Dental Service.

3 A new NHS dental contract was introduced in England and Wales on 1 April 2006. Information based on the new contract are not comparable with information under old contractual arrangements from 1 April 2006 data for England relates to activity under the new contract and cannot be separated between GDS and PDS contracts.

4 Free NHS eye examinations were extended to all on 1st April 2006 in Scotland.

5 Figures refer to patients seen during the 24 month period upto and including the last day of the specified quarters.

Sources: ISD Scotland and Welsh Assembly Government;

The NHS information Centre (IC); 08453006016 , email; enquiries@ic.nhs.uk 


\begin{tabular}{|c|c|c|c|c|c|c|c|c|c|c|c|c|}
\hline \multicolumn{2}{|c|}{ Table 8.7} & \multicolumn{11}{|c|}{ Recorded crime statistics } \\
\hline \multicolumn{12}{|c|}{ England and Wales } & \multirow{2}{*}{$\begin{array}{l}\text { Thousands } \\
\begin{array}{|c}\text { Total } \\
\text { Crime } \\
\text { Offences }\end{array}\end{array}$} \\
\hline & & $\begin{array}{c}\text { Violence } \\
\text { against the } \\
\text { person }\end{array}$ & $\begin{array}{c}\text { Sexual } \\
\text { offences }\end{array}$ & Burglary & Robbery & $\begin{array}{c}\text { Offences } \\
\text { against } \\
\text { vehicles } \\
\end{array}$ & $\begin{array}{c}\text { Other } \\
\text { theft } \\
\text { offences }\end{array}$ & $\begin{array}{c}\text { Fraud and } \\
\text { forgery }\end{array}$ & $\begin{array}{c}\text { Drug } \\
\text { offences }\end{array}$ & $\begin{array}{l}\text { Criminal } \\
\text { damage }\end{array}$ & $\begin{array}{l}\text { Other } \\
\text { Misc }\end{array}$ & \\
\hline \multicolumn{2}{|l|}{$2002 / 03^{1}$} & 845.1 & 58.9 & 890.1 & 110.3 & $1,074.7$ & $1,336.9$ & 331.1 & 143.3 & $1,120.6$ & 64.0 & $5,975.0$ \\
\hline \multicolumn{2}{|c|}{$2003 / 04^{2}$} & 967.2 & 62.5 & 820.0 & 103.7 & 985.0 & $1,327.9$ & 319.6 & 143.5 & $1,218.5$ & 65.7 & $6,013.8$ \\
\hline \multicolumn{2}{|l|}{$2004 / 05^{3}$} & $1,048.1$ & 62.9 & 680.4 & 91.0 & 820.1 & $1,247.6$ & 280.1 & 145.8 & $1,197.5$ & 64.0 & $5,637.5$ \\
\hline \multicolumn{2}{|l|}{$2005 / 06$} & $1,059.6$ & 62.1 & 645.1 & 98.2 & 792.8 & $1,226.2$ & 232.8 & 178.5 & $1,184.3$ & 75.6 & $5,555.2$ \\
\hline \multicolumn{2}{|l|}{$2006 / 07^{4}$} & $1,046.2$ & 57.5 & 622.0 & 101.4 & 765.0 & $1,180.8$ & 199.7 & 194.2 & $1,185.0$ & 75.7 & $5,427.6$ \\
\hline \multicolumn{2}{|l|}{ 2007/08 } & 961.1 & 53.5 & 583.7 & 84.7 & 656.4 & $1,121.0$ & 155.3 & 229.9 & $1,036.2$ & 69.4 & $4,951.2$ \\
\hline \multicolumn{2}{|l|}{ 2008/09 } & 903.5 & 51.4 & 581.6 & 80.1 & 591.8 & $1,080.0$ & 163.2 & 243.5 & 936.4 & 71.2 & $4,702.7$ \\
\hline \multicolumn{2}{|l|}{ 2009/10 } & 871.7 & 54.5 & 540.7 & 75.1 & 495.0 & $1,037.5$ & 152.3 & 235.0 & 806.7 & 70.1 & $4,338.6$ \\
\hline \multirow{5}{*}{2006} & & BEAB & BEAC & BEAD & BEAE & HGW2 & HGW3 & BEAG & LQMO & BEAH & BEAI & BEAA \\
\hline & Q1 & 241.4 & 13.8 & 158.3 & 25.4 & 202.6 & 282.3 & 53.0 & 48.2 & 299.4 & 17.8 & $1,342.2$ \\
\hline & Q2 & 277.3 & 15.7 & 154.3 & 25.4 & 193.9 & 302.2 & 54.4 & 48.3 & 301.2 & 21.0 & $1,393.8$ \\
\hline & Q3 & 276.1 & 15.8 & 152.4 & 24.2 & 185.3 & 305.3 & 53.0 & 46.2 & 281.3 & 19.5 & $1,359.1$ \\
\hline & Q4 & 257.1 & 13.2 & 157.9 & 26.6 & 196.0 & 293.9 & 48.1 & 48.5 & 309.7 & 18.1 & $1,369.2$ \\
\hline \multirow[t]{4}{*}{2007} & Q1 & 235.8 & 12.7 & 157.4 & 25.2 & 189.8 & 279.3 & 44.1 & 51.3 & 292.8 & 17.1 & $1,305.6$ \\
\hline & Q2 & 255.3 & 14.2 & 147.9 & 23.2 & 170.2 & 302.0 & 40.1 & 55.6 & 271.1 & 18.1 & $1,297.6$ \\
\hline & Q3 & 253.0 & 14.4 & 139.9 & 20.0 & 163.6 & 288.1 & 37.8 & 56.4 & 250.0 & 18.0 & $1,241.2$ \\
\hline & Q4 & 230.9 & 12.1 & 145.1 & 21.0 & 159.3 & 271.7 & 36.9 & 58.8 & 256.7 & 16.3 & $1,208.7$ \\
\hline \multirow[t]{4}{*}{2008} & Q1 & 221.1 & 13.0 & 150.8 & 20.6 & 163.4 & 259.3 & 40.7 & 59.2 & 258.6 & 17.1 & $1,204.7$ \\
\hline & Q2 & 236.6 & 14.1 & 138.9 & 19.5 & 152.3 & 287.1 & 44.3 & 60.9 & 243.2 & 18.8 & $1,215.8$ \\
\hline & Q3 & 236.6 & 13.3 & 144.5 & 19.6 & 153.7 & 285.4 & 43.8 & 62.3 & 230.9 & 18.6 & $1,208.6$ \\
\hline & Q4 & 217.5 & 11.8 & 150.8 & 20.6 & 148.2 & 261.0 & 38.0 & 62.5 & 232.3 & 16.8 & $1,159.5$ \\
\hline \multirow[t]{4}{*}{2009} & Q1 & 212.8 & 12.2 & 147.4 & 20.4 & 137.7 & 246.5 & 37.1 & 57.9 & 230.0 & 16.9 & $1,118.8$ \\
\hline & Q2 & 233.0 & 13.9 & 141.7 & 19.6 & 133.8 & 277.8 & 39.5 & 58.6 & 231.6 & 18.3 & $1,167.7$ \\
\hline & Q3 & 226.5 & 13.9 & 133.0 & 17.9 & 123.5 & 270.9 & 38.1 & 59.9 & 206.2 & 18.4 & $1,108.3$ \\
\hline & Q4 & 212.0 & 13.1 & 135.6 & 18.3 & 121.1 & 250.6 & 36.2 & 58.8 & 190.2 & 16.7 & $1,052.7$ \\
\hline \multirow[t]{2}{*}{2010} & Q1 & 200.0 & 13.5 & 130.4 & 19.3 & 116.5 & 238.2 & 38.4 & 58.3 & 178.6 & 16.7 & $1,009.9$ \\
\hline & Q2 & 224.4 & 14.7 & 129.5 & 19.4 & 118.1 & 282.3 & 39.0 & 58.1 & 193.4 & 18.6 & $1,097.6$ \\
\hline
\end{tabular}

Note: Financial year data is only revised annually in July of each year.

1 The National Crime Recording Standard (NCRS) was introduced across all police forces from April 2002, therefore figures before and after that date are not directly comparable. The introduction of the NCRS has increased the recorded crime figures significantly. For 2002/03 it is estimated

that the implementation of the Standard inflated the total number of recorded crimes by $10 \%$ in its first year, although the impact differs for each offence group.Violence against the person was particularly affected.

2 Much of the increase in violence against the person in 2003/04 is likely to be due to the continuing impact of changes in recording.

3 The Sexual Offences Act 2003, introduced in May 2004, re-defined many sexual offences. This change in legislation could, in itself, account for

much of the increase in recorded sexual offences and means figures for 2004/05 are not comparable with earlier years.

4 The Fraud Act 2006 commenced on 15 January 2007, altering the definition and coverage of fraud and forgery offences. At that time, the counting of cheque and credit card fraud changed to a 'per account' basis rather than a 'per transaction' basis.

Source: Home Office: 02070350307

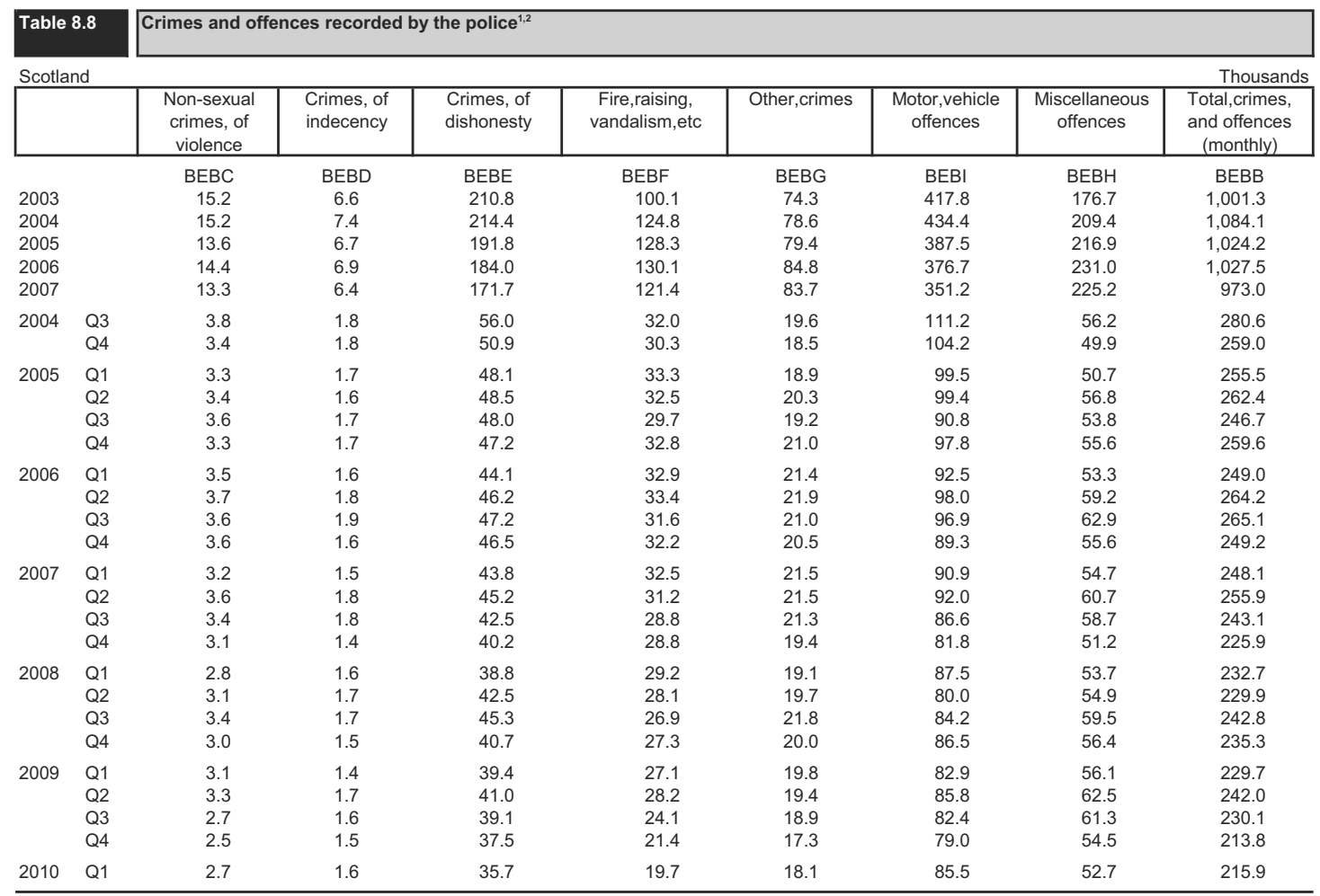

1 Components may not add to totals due to separate rounding.

2 The introduction of the Scottish Crime recording Standard on 1 April 2004 has increased the number of minor crimes recorded, such as minor crimes of vandalism and petty thefts.

Source: The Scottish Government Justice Department: 01312442635 


\section{Table 8.9}

Telephones

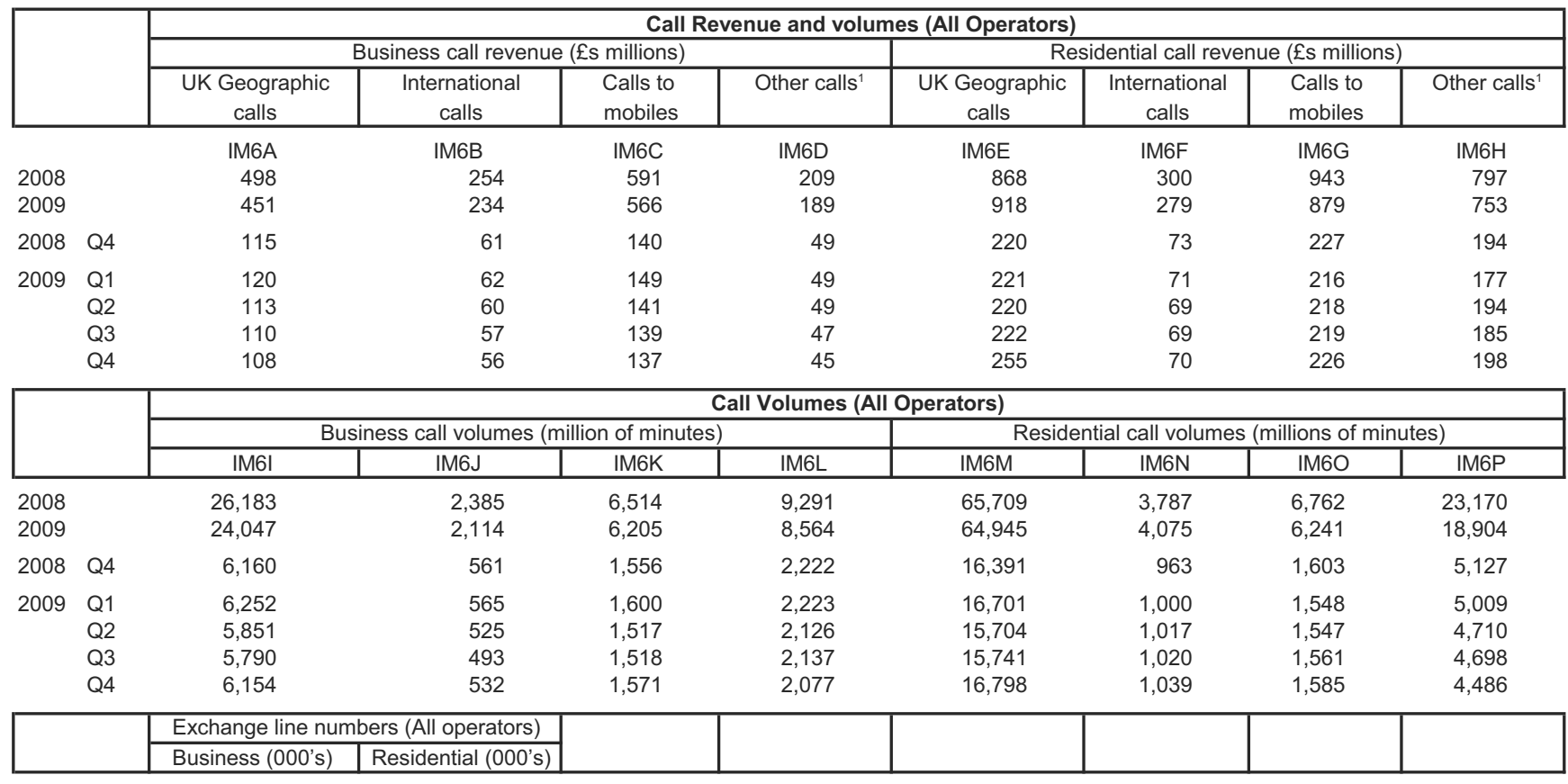

$\begin{array}{rrrr} & & \text { IM6Q } & \text { IM6R } \\ 2008 & & 9,710 & 23,525 \\ 2009 & & 9,223 & 22,894 \\ 2008 & \text { Q4 } & 9,710 & 23,525 \\ 2009 & \text { Q1 } & 9,666 & 23,459 \\ & \text { Q2 } & 9,399 & 23,388 \\ & \text { Q3 } & 9,311 & 23,216 \\ & \text { Q4 } & 9,223 & 22,894\end{array}$

1 Includes freephone, special services, premium rate, directory enquiries and all other call types.

Source: Ofcom Tel: 02079813000 


\begin{tabular}{|c|c|c|c|c|c|c|c|}
\hline \multirow[t]{2}{*}{ Radio Listening } & \multicolumn{7}{|c|}{ Radio Listening } \\
\hline & $\begin{array}{c}\text { Adult }(15+) \\
\text { Population '000`s }\end{array}$ & $\begin{array}{c}\text { Weekly } \\
\text { Reach '000‘s }\end{array}$ & $\begin{array}{c}\text { Weekly } \\
\text { Reach \% }\end{array}$ & $\begin{array}{c}\text { Average } \\
\text { Hours } \\
\text { Per Head }\end{array}$ & $\begin{array}{l}\text { Average Hours } \\
\text { Per Listener }\end{array}$ & $\begin{array}{c}\text { Total } \\
\text { Hours (000's) }\end{array}$ & $\begin{array}{l}\text { Share of } \\
\text { Listening }\end{array}$ \\
\hline \multicolumn{8}{|c|}{ Quarterly Summary of Radio Listening -Period Sept 2010} \\
\hline All Radio & 51,618 & 46,762 & 90.6 & 20.4 & 22.6 & $1,055,210$ & 100.0 \\
\hline All Commercial Radio & 51,618 & 33,373 & 64.7 & 8.9 & 13.7 & 458,133 & 43.4 \\
\hline All National Commercial & 51,618 & 15,101 & 29.3 & 2.3 & 7.9 & 118,566 & 11.2 \\
\hline All Local Commercial & 51,618 & 26,999 & 52.3 & 6.6 & 12.6 & 339,567 & 32.2 \\
\hline All BBC & 51,618 & 34,243 & 66.3 & 11.1 & 16.7 & 573,459 & 54.3 \\
\hline All BBC Network Radio & 51,618 & 31,018 & 60.1 & 9.3 & 15.4 & 478,876 & 45.4 \\
\hline BBC Local/Regional & 51,618 & 9,144 & 17.7 & 1.8 & 10.3 & 94,584 & 9.0 \\
\hline BBC Radio 1 & 51,618 & 11,647 & 22.6 & 1.9 & 8.2 & 95,729 & 9.1 \\
\hline BBC Radio 2 & 51,618 & 13,682 & 26.5 & 3.2 & 12.0 & 164,809 & 15.6 \\
\hline BBC Radio 3 & 51,618 & 2,145 & 4.2 & 0.2 & 6.0 & 12,776 & 1.2 \\
\hline BBC Radio 4 & 51,618 & 10,368 & 20.1 & 2.4 & 12.0 & 124,051 & 11.8 \\
\hline BBC Radio 5 Live & 51,618 & 6,295 & 12.2 & 1.0 & 7.9 & 49,574 & 4.7 \\
\hline FIVE live sports extra & 51,618 & 648 & 1.3 & 0.0 & 2.8 & 1,784 & 0.2 \\
\hline BBC 6 Music & 51,618 & 1,196 & 2.3 & 0.2 & 8.6 & 10,339 & 1.0 \\
\hline BBC Radio7 & 51,618 & 1,045 & 2.0 & 0.1 & 6.4 & 6,691 & 0.6 \\
\hline BBC Asian Network UK & 51,618 & 462 & 0.9 & 0.0 & 5.1 & 2,350 & 0.2 \\
\hline BBC World Service & 51,618 & 1,319 & 2.6 & 0.1 & 4.7 & 6,216 & 0.6 \\
\hline $1 \mathrm{Xtra}$ from the $\mathrm{BBC}$ & 51,618 & 806 & 1.6 & 0.1 & 5.7 & 4,556 & 0.4 \\
\hline \multicolumn{8}{|l|}{$\begin{array}{l}\text { Radio Listening (UK): Average Weekly } \\
\text { Average hours per head (by age group). } \\
\text { Period ending Sept } 2010\end{array}$} \\
\hline Age 4-14 & 9.7 & & & & & & \\
\hline Age $15-24$ & 15.3 & & & & & & \\
\hline Age $25-34$ & 17.5 & & & & & & \\
\hline Age $35-44$ & 20.7 & & & & & & \\
\hline Age $45-54$ & 22.0 & & & & & & \\
\hline Age $55-64$ & 23.7 & & & & & & \\
\hline Age 65 and Over & 23.0 & & & & & & \\
\hline Overall Average Weekly Listening Hours (4+) & 19.7 & & & & & & \\
\hline
\end{tabular}

Source: RAJAR / IpsosMori / RSMB 


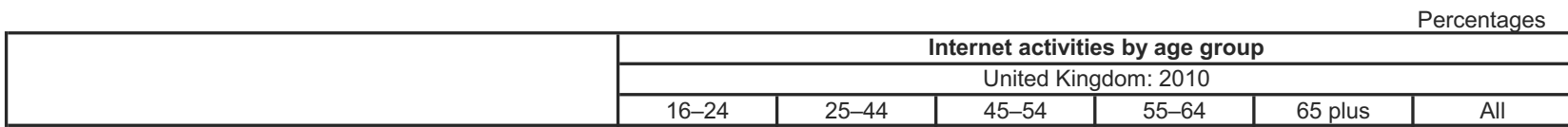

Finding information about goods or services Sending/receiving emails

Using services related to travel and accommodation Internet banking

Looking for information-education, training, courses

Reading or downloading online news, magazines

Looking for a job or sending job application

Seeking health-related infor mation

Downloading software (other than games)

Selling of goods or services (eg. via auctions)

Consulting the Internet with the purpose of learning

Playing or downloading games, images, films or music

Listening to web radio or watching web TV

Uploading self created content

Posting messages to chat sites, blogs, newsgroups etc

Telephoning over the Internet/video calls (via webcam)

Doing an online course

Donating to charities online

\section{4}

88

50

45

47

52

38

27

35

16

47

61

59

50

75

30

11

10

80

$\begin{array}{rrr}83 & 72 & 75 \\ 91 & 87 & 90 \\ 72 & 62 & 63 \\ 53 & 34 & 54 \\ 19 & 7 & 32 \\ 47 & 40 & 51 \\ 11 & 1 & 26 \\ 44 & 36 & 39 \\ 27 & 18 & 30 \\ 18 & 9 & 21 \\ 30 & 27 & 35 \\ 24 & 17 & 40 \\ 34 & 24 & 45 \\ 29 & 22 & 38 \\ 19 & 8 & 43 \\ 17 & 15 & 23 \\ 5 & 3 & 8 \\ 13 & 7 & 12\end{array}$

$76 \quad 80$

90

$\begin{array}{ll}80 & 91\end{array}$

$\begin{array}{ll}70 & 72\end{array}$

$\begin{array}{ll}70 & 72 \\ 54 & 53\end{array}$

$\begin{array}{ll}54 & 53 \\ 27 & 19\end{array}$

$\begin{array}{ll}51 & 47\end{array}$

$\begin{array}{ll}51 & 47 \\ 23 & 11\end{array}$

$\begin{array}{ll}39 & 44\end{array}$

$\begin{array}{ll}23 & 27 \\ 20 & 18\end{array}$

$\begin{array}{ll}20 & 18 \\ 34 & 30\end{array}$

$\begin{array}{ll}32 & 24\end{array}$

$45 \quad 34$

$\begin{array}{ll}28 & 29 \\ 31 & 19\end{array}$

$\begin{array}{ll}31 & 19 \\ 22 & 17\end{array}$

$\begin{array}{rr}7 & 13\end{array}$

\begin{tabular}{|c|c|c|c|c|c|c|}
\hline & \multicolumn{6}{|c|}{ Internet purchases by age group: United Kingdom } \\
\hline & $16-24$ & $25-44$ & $45-54$ & $55-64$ & 65 plus & All \\
\hline Holiday, accommodation (eg hotels) & 46 & 53 & 54 & 47 & 44 & 44 \\
\hline Films, music & 50 & 50 & 33 & 27 & 25 & 47 \\
\hline Clothes, sports goods & 58 & 45 & 46 & 43 & 38 & 52 \\
\hline Household goods (eg furniture, toys, etc) & 52 & 41 & 46 & 39 & 37 & 43 \\
\hline Books, magazines or newspapers (including e-books) & 40 & 43 & 39 & 41 & 40 & 39 \\
\hline Other travel arrangements (eg transport tickets, car hire) & 36 & 42 & 48 & 35 & 32 & 36 \\
\hline Tickets for events & 37 & 40 & 34 & 21 & 19 & 35 \\
\hline Electronic equipment (including cameras) & 31 & 24 & 22 & 14 & 15 & 25 \\
\hline Video games software and upgrades & 30 & 20 & 9 & 8 & 8 & 23 \\
\hline Computer software and upgrades & 18 & 17 & 23 & 22 & 20 & 18 \\
\hline Food and groceries & 32 & 25 & 16 & 17 & 17 & 24 \\
\hline Computer Hardware & 13 & 15 & 14 & 11 & 10 & 13 \\
\hline Shares, financial services or insurance & 24 & 23 & 18 & 13 & 11 & 20 \\
\hline e-learning material & 8 & 5 & 2 & 2 & 2 & 6 \\
\hline Medicine & 6 & 6 & 9 & 12 & 10 & 6 \\
\hline Telecommunication services & 17 & 14 & 16 & 13 & 13 & 15 \\
\hline Other & 4 & 6 & 7 & 5 & 5 & 5 \\
\hline
\end{tabular}

\begin{tabular}{|l|c|c|c|}
\hline \multirow{3}{*}{ Year } & \multicolumn{3}{|c|}{ Households with access to the Internet 2006 to 2010 } \\
\cline { 2 - 4 } & Per cent & $\begin{array}{c}\text { Internet access } \\
\text { households (millions) }\end{array}$ & $\begin{array}{c}\text { Percentage change on } \\
\text { previous year }\end{array}$ \\
\cline { 2 - 4 } & 57 & 14.3 &. \\
2006 & 61 & 15.2 & 7 \\
2007 & 65 & 16.5 & 8 \\
2008 & 70 & 18.3 & 11 \\
2009 & 73 & 19.2 & 5 \\
2010 & 53 & \multicolumn{3}{|c|}{} \\
\hline
\end{tabular}

Source: Statistical Bulletin 2010, Office for National Statistics 


\begin{tabular}{|c|c|c|c|c|c|c|c|c|c|}
\hline & & $\begin{array}{c}\text { Price of first } \\
\text { class stamp }(p)\end{array}$ & $\begin{array}{l}\text { Volume of first } \\
\text { class stamped } \\
\text { mail delivered } \\
\text { (million items) }\end{array}$ & $\begin{array}{c}\text { Total first } \\
\text { class mail } \\
\text { delivered } \\
\text { (million items) }\end{array}$ & $\begin{array}{l}\text { Price of second } \\
\text { class stamp }(p)\end{array}$ & $\begin{array}{l}\text { Volume of second } \\
\text { class stamped } \\
\text { mail delivered } \\
\text { (million items) }\end{array}$ & $\begin{array}{c}\text { Total second } \\
\text { class mail } \\
\text { delivered } \\
\text { (million items) }\end{array}$ & $\begin{array}{l}\text { Domestic } \\
\text { (million) }\end{array}$ & $\begin{array}{c}\text { International } \\
\text { (million) }\end{array}$ \\
\hline \multirow{5}{*}{2006} & & IM7A & IM7B & IM7C & IM7D & IM7E & IM7F & IM7G & IM7H \\
\hline & Q1 & 30 & 399 & 1,280 & 21 & 284 & 2,059 & 8.6 & 0.5 \\
\hline & Q2 & 32 & 396 & 1,292 & 23 & 267 & 1,978 & 9.0 & 0.6 \\
\hline & Q3 & 32 & 340 & 1,217 & 23 & 242 & 1,811 & 9.0 & 0.5 \\
\hline & Q4 & 32 & 479 & 1,377 & 23 & 507 & 2,146 & 11.8 & 0.8 \\
\hline \multirow[t]{4}{*}{2007} & Q1 & 32 & 365 & 1,189 & 23 & 272 & 1,786 & 9.6 & 0.6 \\
\hline & Q2 & 34 & 342 & 1,185 & 24 & 244 & 1,696 & 11.1 & 0.6 \\
\hline & Q3 & 34 & 320 & 1,118 & 24 & 229 & 1,560 & 9.8 & 0.6 \\
\hline & Q4 & 34 & 412 & 1,250 & 24 & 457 & 1,852 & 12.4 & 0.8 \\
\hline \multirow[t]{4}{*}{2008} & Q1 & 34 & 373 & 1,195 & 24 & 282 & 1,664 & 10.5 & 0.7 \\
\hline & Q2 & 36 & 317 & 1,092 & 27 & 238 & 1,490 & 10.8 & 0.7 \\
\hline & Q3 & 36 & 259 & 997 & 27 & 205 & 1,362 & 9.7 & 0.9 \\
\hline & Q4 & 36 & 351 & 1,092 & 27 & 433 & 1,580 & 12.1 & 1.0 \\
\hline \multirow[t]{4}{*}{2009} & Q1 & 36 & 295 & 974 & 27 & 250 & 1,399 & 10.4 & 0.9 \\
\hline & Q2 & 39 & 291 & 965 & 30 & 223 & 1,285 & 11.5 & 0.3 \\
\hline & Q3 & 39 & 232 & 856 & 30 & 183 & 1,118 & 10.6 & 0.3 \\
\hline & Q4 & 39 & 307 & 936 & 30 & 379 & 1,279 & 12.1 & 1.1 \\
\hline \multirow[t]{3}{*}{2010} & Q1 & 39 & 282 & 849 & 30 & 231 & 1,103 & 10.5 & 0.9 \\
\hline & Q2 & 41 & 216 & 822 & 32 & 165 & 1,009 & 11.9 & 0.9 \\
\hline & Q3 & 41 & 222 & 769 & 32 & 168 & 952 & 12.4 & 0.9 \\
\hline
\end{tabular}

\begin{tabular}{|c|c|c|c|c|c|c|c|c|c|c|c|c|c|c|c|}
\hline & & 1996 & 1997 & 1998 & 1999 & 2000 & 2001 & 2002 & 2003 & 2004 & 2005 & 2006 & 2007 & 2008 & 2009 \\
\hline $\begin{array}{l}\text { Letters, etc posted (millions) } \\
\text { of which: }\end{array}$ & KMRA & 18,101 & 18,350 & 18,878 & 24,880 & 24,089 & 23,705 & 20,648 & 21,979 & 22,837 & 24,341 & 24,880 & 24,089 & 23,705 & .. \\
\hline gistered and insured & KMRB & 25.6 & 28.7 & 31.6 & 30.2 & 32.3 & 36.1 & 38.5 & 41.4 & 45.3 & 45.3 & 45.3 & 44.7 & 46.6 &.. \\
\hline irmail (Commonwealth and foreign) & KMRC & 684.5 & 658.4 & 693.2 & 672.3 & 659.2 & 600.7 & 541.6 & 512.0 & 457.9 & 502.2 & 502.2 & 541.0 & 470.3 & .. \\
\hline usiness reply and freepost items & KMRD & 505.8 & 524.7 & 503.6 & 475.3 & 487.4 & 486.2 & 434.4 & 397.7 & 401.1 & 402.3 & 402.3 & 373.7 & 349.1 & .. \\
\hline th & & & & & & & & & & & & & & & \\
\hline otal issued (thousands) ${ }^{3}$ & KMRH & 33,404 & 31,907 & 30,289 & 30,289 & 30,153 & 30,931 & 29,150 & 28,666 & 28,888 & 29,344 & 20,489 & 19,714 & 16,650 & .. \\
\hline
\end{tabular}

Total issued (thousands)

1 "Volume of first class stamped mail delivered" and "volume of second stamped mail delivered" relates to stamped mail only.

2 "Total first class mail delivered" and "total second class mail delivered" relates to all first and second class products (ie not just stamped mail) This includes first and second class customer sorted mail ( Mailsort, Press Stream and Walksort products -where customers pre-sort mail that is to be delivered).

3 Excluding those issued on HM ships, in many British possessions and in other places abroad. Up to 1998 includes Postal Orders issued Overseas and by Ministry of Defence.

Sources: Royal Mail: 0207250 2633;

Capita Business Services: 01173021003

Post Office Limited: 02073207424 


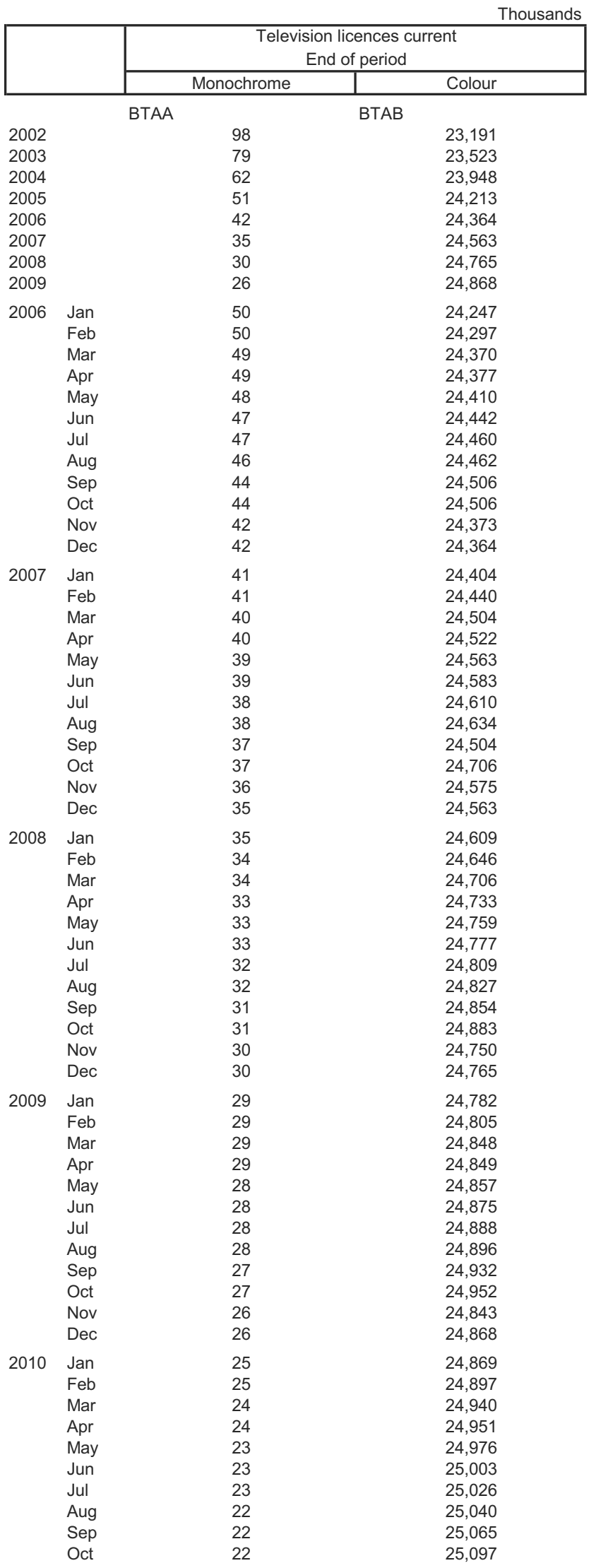

1 Not sales figures, but are the amount of 'Licences in Force' - i.e. how many licences are valid at that point in time.

Source: Capita Business Services Limited: 01173021003 


\begin{tabular}{|c|c|c|c|c|c|c|c|c|c|c|c|c|c|c|c|c|}
\hline Table 8.14 & 5 of & ge I & le $R$ & ders & of & tion & I daily n & newspa & apers & & & & & & & \\
\hline 12 months' & & & & & & & & & & & & & & & & Tho \\
\hline & & & 20 & & & & & 08 & & & 20 & 09 & & & 2010 & \\
\hline & & Mar & Jun & Sep & Dec & Mar & Jun & Sep & $\mathrm{Dec}$ & Mar & Jun & Sep & Dec & Mar & Jun & Sep \\
\hline The Sun & WSDV & 7,840 & 7,768 & 7,931 & 7,980 & 7,897 & 8,031 & 7,949 & 7,872 & 7,870 & 7,860 & 7,798 & 7,761 & 7,751 & 7,694 & 7,700 \\
\hline Daily Mail & WSEI & 5,253 & 5,197 & 5,239 & 5,230 & 5,293 & 5,347 & 5,212 & 5,062 & 4,949 & 4,846 & 4,936 & 4,934 & 4,881 & 4,896 & 4,739 \\
\hline Daily Mirror/Daily Record & WSEH & 4,937 & 4,975 & 4,971 & 4,895 & 4,904 & 4,864 & 4,758 & 4,717 & 4,555 & 4,608 & 4,476 & 4,404 & 4,288 & 4,170 & 4,004 \\
\hline Daily Mirror & WSEM & 3,844 & 3,880 & 3,868 & 3,789 & 3,748 & 3,685 & 3,623 & 3,600 & 3,489 & 3,566 & 3,477 & 3,425 & 3,381 & 3,244 & 3,117 \\
\hline The Daily Telegraph & WSEN & 2,177 & 2,167 & 2,054 & 2,075 & 2,023 & 2,060 & 2,048 & 1,901 & 1,887 & 1,843 & 1,834 & 1,905 & 1,840 & 1,796 & 1,751 \\
\hline The Times & WSES & 1,730 & 1,702 & 1,672 & 1,666 & 1,673 & 1,731 & 1,764 & 1,813 & 1,770 & 1,801 & 1,802 & 1,773 & 1,768 & 1,673 & 1,613 \\
\hline Daily Star & WSEQ & 1,620 & 1,701 & 1,690 & 1,597 & 1,500 & 1,484 & 1,417 & 1,427 & 1,451 & 1,471 & 1,546 & 1,577 & 1,617 & 1,586 & 1,551 \\
\hline Daily Express & WSEP & 1,742 & 1,694 & 1,687 & 1,678 & 1,621 & 1,598 & 1,605 & 1,571 & 1,557 & 1,624 & 1,598 & 1,577 & 1,529 & 1,463 & 1,423 \\
\hline The Guardian & WSET & 1,239 & 1,226 & 1,193 & 1,121 & 1,169 & 1,165 & 1,240 & 1,240 & 1,206 & 1,205 & 1,142 & 1,147 & 1,124 & 1,132 & 1,130 \\
\hline The Independent & WSEU & 767 & 774 & 787 & 745 & 733 & 702 & 722 & 688 & 649 & 679 & 636 & 671 & 635 & 607 & 556 \\
\hline Financial Times & WSEY & 394 & 398 & 375 & 360 & 362 & 377 & 387 & 418 & 417 & 430 & 433 & 434 & 418 & 404 & 391 \\
\hline Any national morning & WSEZ & 21,782 & 21,702 & 21,709 & 21,650 & 21,536 & 21,625 & 21,475 & 21,203 & 20,918 & 20,817 & 20,609 & 20,574 & 20,404 & 20,207 & 19,841 \\
\hline
\end{tabular}

Readership Surveys Ltd.

\begin{tabular}{|c|c|c|c|c|c|c|}
\hline \multicolumn{2}{|c|}{ Table 8.15} & \multicolumn{5}{|c|}{ Overseas travel and tourism ${ }^{1}$} \\
\hline & & & & & & Not seasonally adjusted \\
\hline & & $\begin{array}{l}\text { Visits by overseas } \\
\text { visitors to the UK } \\
\text { (thousands) }\end{array}$ & $\begin{array}{c}\text { Expenditure by overseas } \\
\text { visitors to the UK } \\
\text { ( } £ \text { million) }\end{array}$ & $\begin{array}{l}\text { Visits by UK } \\
\text { residents abroad } \\
\text { (thousands) }\end{array}$ & $\begin{array}{c}\text { Expenditure by } \\
\text { UK residents abroad } \\
\text { (£ million) }\end{array}$ & $\begin{array}{l}\text { Net earnings in UK } \\
(£ \text { million })\end{array}$ \\
\hline & & GMAA & GMAK & GMAF & GMAM & GMAO \\
\hline 2004 & & 27755.0 & 13047.0 & 64194.0 & 30285.0 & -17238.0 \\
\hline 2005 & & 29970.0 & 14248.0 & 66441.0 & 32154.0 & -17906.0 \\
\hline 2006 & & 32713.0 & 16002.0 & 69536.0 & 34411.0 & -18409.0 \\
\hline 2007 & & 32778.0 & 15960.0 & 69450.0 & 35013.0 & -19053.0 \\
\hline 2008 & & 31888.0 & 16326.0 & 69008.0 & 36837.0 & .. \\
\hline 2009 & & 29892.0 & 16592.0 & 58614.0 & 31695.0 & .. \\
\hline \multirow[t]{4}{*}{2004} & Q1 & 5,451 & 2,229 & 11,770 & 5,730 & $-3,500$ \\
\hline & Q2 & 7,015 & 3,232 & 16,967 & 7,602 & $-4,370$ \\
\hline & Q3 & 8,412 & 4,390 & 21,233 & 10,437 & $-6,047$ \\
\hline & Q4 & 6,748 & 3,196 & 14,204 & 6,516 & $-3,321$ \\
\hline \multirow[t]{4}{*}{2005} & Q1 & 6,171 & 2,643 & 12,821 & 6,412 & $-3,767$ \\
\hline & Q2 & 7,868 & 3,562 & 17,417 & 8,110 & $-4,548$ \\
\hline & Q3 & 8,858 & 4,474 & 21,767 & 10,911 & $-6,438$ \\
\hline & Q4 & 7,073 & 3,568 & 14,437 & 6,722 & $-3,154$ \\
\hline \multirow[t]{4}{*}{2006} & Q1 & 6,351 & 2,757 & 13,130 & 6,665 & $-3,907$ \\
\hline & Q2 & 8,476 & 3,970 & 18,738 & 8,765 & $-4,795$ \\
\hline & Q3 & 10,296 & 5,401 & 22,378 & 11,543 & $-6,142$ \\
\hline & Q4 & 7,592 & 3,873 & 15,289 & 7,438 & $-3,565$ \\
\hline \multirow[t]{4}{*}{2007} & Q1 & 6,737 & 3,000 & 13,495 & 6,908 & $-3,907$ \\
\hline & Q2 & 8,511 & 3,859 & 17,976 & 8,834 & $-4,975$ \\
\hline & Q3 & 9,564 & 5,234 & 23,000 & 12,097 & $-6,863$ \\
\hline & Q4 & 7,966 & 3,866 & 14,978 & 7,174 & $-3,308$ \\
\hline \multirow[t]{4}{*}{2008} & Q1 & 7,195 & 3,130 & 14,362 & 7,797 & $-4,633$ \\
\hline & Q2 & 8,406 & 4,232 & 18,548 & 9,312 & $-4,963$ \\
\hline & Q3 & 9,358 & 5,195 & 22,467 & 12,758 & $-7,422$ \\
\hline & Q4 & 6,929 & 3,769 & 13,631 & 6,970 & .. \\
\hline \multirow[t]{4}{*}{2009} & Q1 & 6,213 & 3,221 & 11,286 & 6,763 & .. \\
\hline & Q2 & 7,943 & 4,097 & 15,961 & 8,020 & .. \\
\hline & Q3 & 8,779 & 5,418 & 19,775 & 10,944 & .. \\
\hline & Q4 & 6,951 & 3,855 & 11,592 & 5,968 & .. \\
\hline \multirow[t]{3}{*}{2010} & Q1 & 5,905 & 2,937 & 10,150 & 6,047 & .. \\
\hline & Q2 & 7,707 & 4,303 & 14,495 & 7,920 & .. \\
\hline & Q3 & 8,888 & 5,299 & 19,186 & 11,017 & .. \\
\hline
\end{tabular}

1 Due to rounding methodology, quarterly figures will not always sum to the annual total.

Source: International Passenger Survey, Office for National Statistics 


\begin{tabular}{|c|c|c|c|c|c|c|c|c|c|c|}
\hline & \multicolumn{10}{|c|}{ Air temperature (degrees celsius) } \\
\hline & \multirow{2}{*}{$\begin{array}{c}\text { Highest } \\
\text { maximum }^{2}\end{array}$} & \multirow{2}{*}{$\begin{array}{c}\text { Lowest } \\
\text { minimum }^{2}\end{array}$} & \multicolumn{3}{|c|}{ Difference from average } & \multicolumn{2}{|c|}{ Difference from average } & \multicolumn{2}{|c|}{ Percent of average } & \multirow{2}{*}{$\begin{array}{c}\text { Sunshine } \\
\text { (hours) }\end{array}$} \\
\hline & & & Maximum & Minimum & Mean & $\begin{array}{c}\text { Mean } 30 \mathrm{~cm} \text { soil } \\
\text { temperature } \\
\text { (degrees celsius) }\end{array}$ & Raindays $^{3}$ & Rainfall & Sunshine & \\
\hline \multicolumn{11}{|l|}{ May 2010} \\
\hline 0 Scotland $\mathrm{N}$ & 24.0 & -6.0 & 0.0 & -0.4 & -0.2 & 1.1 & 3 & 67 & 112 & 178.2 \\
\hline 1 Scotland $\mathrm{E}$ & 27.0 & -6.0 & 0.6 & -0.4 & 0.1 & 1.1 & 1 & 62 & 118 & 193.1 \\
\hline 2 England $\mathrm{E} \& \mathrm{NE}$ & 27.9 & -5.7 & 0.4 & -0.3 & 0.1 & 0.8 & -3 & 39 & 118 & 210.8 \\
\hline 3 East Anglia & 28.4 & -3.2 & 0.0 & -0.7 & -0.4 & 0.4 & -4 & 54 & 110 & 213.0 \\
\hline 4 Midlands & 28.9 & -2.7 & 0.4 & -0.1 & 0.1 & 0.9 & -3 & 53 & 111 & 198.1 \\
\hline 5 England SE & 28.8 & -3.0 & 0.2 & -0.8 & -0.3 & 0.2 & -4 & 54 & 102 & 203.0 \\
\hline 6 Scotland W & 25.8 & -4.5 & 0.5 & -0.4 & 0.0 & 1.0 & -1 & 43 & 116 & 205.9 \\
\hline 7 England NW \& N Wales & 27.2 & -5.8 & 0.2 & -0.4 & -0.1 & 0.9 & -2 & 48 & 111 & 201.4 \\
\hline 8 England SW \& S Wales & 27.2 & -4.3 & 0.6 & -0.3 & 0.2 & 0.6 & -1 & 59 & 108 & 207.0 \\
\hline $\mathrm{N}$ Ireland & 26.2 & -2.8 & 0.7 & 0.0 & 0.3 & 1.1 & -1 & 59 & 115 & 199.5 \\
\hline Scotland & 27.0 & -6.0 & 0.4 & -0.4 & 0.0 & 1.1 & 1 & 58 & 115 & 191.1 \\
\hline England & 28.9 & -5.8 & 0.3 & -0.4 & 0.0 & 0.6 & -3 & 49 & 109 & 203.3 \\
\hline Wales & 27.2 & -4.3 & 0.5 & -0.4 & 0.1 & 0.9 & 0 & 62 & 117 & 214.5 \\
\hline England \& Wales & 28.9 & -5.8 & 0.3 & -0.4 & 0.0 & 0.7 & -3 & 52 & 110 & 204.8 \\
\hline
\end{tabular}

Note: Anomalies are with respect to the 1961-90 averaging period. However, anomalies with respect to the 1971-2000 averaging period are available from http://www.metoffice.gov.uk/climate/uk

The first half of May was characterised by cool weather, with a north-easterly or northerly airstream. There were showers, especialy early on and over Scotland and Northern Ireland, and some frosts. Temperatures recovered during the second half of the month, with a very warm spell from 20th to 24, when some new temperature records for May were set in Scotland. Mean temperatures for the month ended up close, or slightly below, to normal. May saw below average rainfall across almost all of the UK, with less than $50 \%$ of normal in much of western Scotland and central southern England and in a broad swathe from East Anglia to Cumbria It was the driest May across the UK since 1991. Sunshine totals were close to, or a little above, normal.

1 District values for each element are computed using all available climate stations, excluding rooftop sites for minimum air temperature. The values in the table may not be compatible with other time series (eg. Central England Temperature, England and Wales Rainfall).

2 Highest maximum and lowest minimum air temperatures for each district are recorded values at individual weather stations.

3 Raindays are the number of days during which the total precipitation is at least $0.2 \mathrm{~mm}$.

Source: Met Office 